\title{
Ultra-Performance Liquid Chromatography-Q- Exactive Orbitrap-Mass Spectrometry Analysis for Metabolic Communication between Heart and Kidney in Adriamycin-Induced Nephropathy Rats
}

\author{
Chunliu Wanga,b Jiping Liang ${ }^{\mathrm{a}}$ Wenwen Yang ${ }^{\mathrm{a}}$ Shixiang Wang ${ }^{\mathrm{a}} \quad \mathrm{Jie}_{\mathrm{Yu}}^{\mathrm{a}}$ \\ Pu Jia ${ }^{a}$ Yapeng $\mathrm{Du}^{\mathrm{a}}$ Mei Wang ${ }^{\mathrm{a}}{\text { Ye } \mathrm{Li}^{\mathrm{b}} \text { Xiaohui Zheng }}^{\mathrm{a}}$

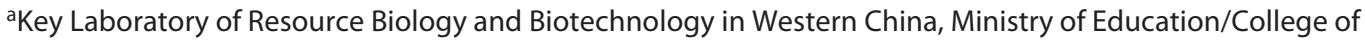 \\ Life Science, Northwest University, Xi'an, China; 'bnstitute of Traditional Chinese Medicine, Shaanxi Academy of \\ Traditional Chinese Medicine, Xi'an, China
}

\section{Keywords}

Adriamycin-induced nephropathy - Cardiorenal metabolic communication - Metabolomics · Alanine - Aspartate and glutamate metabolism pathway $\cdot$ Succinic acid

\begin{abstract}
Background/Aims: Although the adriamycin-induced nephropathy model is frequently employed in the study of nephrotic syndrome and focal segmental glomerulosclerosis, the accompanying myocardial damage has always been a cause for concern. Therefore, there is a great need to study cardiorenal communication in this model. Methods: An adriamycin-induced nephropathy model was established via tail vein injection. The levels of the biochemical indicators serum albumin, serum globulin, serum total protein, serum cholesterol, serum creatinine ( $\mathrm{SCr}$ ), urinary protein, and urinary creatinine $(\mathrm{UCr}$ ) were measured, and histopathological changes in the heart and kidneys were assessed using hematoxylineosin staining. Metabolomic changes in the heart, blood, and kidneys were analyzed using the metabolomics method based on ultra-performance liquid chromatography Q-Exactive Orbitrap mass spectrometry. Results: Compared with
\end{abstract}

karger@karger.com www.karger.com/kbr

Karger $\stackrel{\text { ' }}{5}$

GOPEN ACCESS
(C) 2021 The Author(s)

Published by S. Karger AG, Basel

This is an Open Access article licensed under the Creative Commons Attribution-NonCommercial-4.0 International License (CC BY-NC) (http://www.karger.com/Services/OpenAccessLicense), applicable to the online version of the article only. Usage and distribution for commercial purposes requires written permission. the control group, the model group showed significant decreases in serum protein and total protein levels, albumin/ globulin ratio, and creatinine clearance rate as well as significant increases in serum cholesterol, $\mathrm{SCr}$, urinary protein, and $\mathrm{UCr}$ levels. Significant pathological changes were observed in the renal pathology sections in the model group, including diffusely merged glomerular epithelial cells, inflammatory infiltration, and vacuolated glomerular cells. Additionally, thickened myocardial fibers, swollen nuclei, inflammatory infiltration, and partial myocardial necrosis could be seen in the cardiac pathology sections in the model group. Based on multivariate statistical analysis, a total of 20 differential metabolites associated with 15 metabolic pathways were identified in the heart, 7 differential metabolites with 7 metabolic pathways were identified in the blood, and 16 differential metabolites with 21 metabolic pathways were identified in the kidney. Moreover, 6 common metabolic pathways shared by the heart and kidney were identified: arginine and proline metabolism; arginine biosynthesis; glutathione metabolism; alanine, aspartate, and glutamate metabolism; beta-alanine metabolism; and histidine metabolism. Among these metabolic pathways, alanine, aspartate, and glutamate metabolism was shared by the heart, blood,
Correspondence to:

Pu Jia, jiapu77@126.com

Ye Li, liyelsj@163.com

Xiaohui Zheng, zhengxh318@ nwu.edu.cn 
and kidney. Succinic acid was found to be the key regulatory metabolite in cardiorenal metabolic communication. Conclusion: Six metabolic pathways were found to be involved in cardiorenal metabolic communication in an adriamycininduced nephropathy model, in which alanine, aspartate, and glutamate metabolism may be the metabolic link between the heart and kidney in the development and maintenance of oxidative stress and inflammation. Succinic acid may serve as a key regulatory metabolic switch or marker of cardiac and renal co-injury, as shown in an adriamycin-induced nephropathy model.

(C) 2021 The Author(s)

Published by S. Karger AG, Basel

\section{Introduction}

Adriamycin is an anthracycline antibiotic that exerts anticancer effects by inhibiting DNA and RNA synthesis. However, a side effect of nephrotoxicity was primarily reported in the clinical setting. Since it was first introduced by Bertani et al. [1], adriamycin-induced nephropathy has been frequently used as an animal model of nephrotic syndrome (NS) and focal segmental glomerulosclerosis in recent years [2-4]. The model is stable and easy to operate. Nephropathy can be induced via a single intravenous administration. Overt proteinuria, hypoalbuminemia, and hyperlipidemia are the most frequent distinguishing features.

Renal neoplasms form with various magnitudes of glomerular cells, and glomerulosclerosis emerges after tubulointerstitial lesions [2]. Although the adriamycininduced nephropathy model is similar to clinical focal segmental glomerulosclerosis in terms of renal pathological development, the side effects, particularly heart damage, have been a cause of concern. Following intravenous administration, some amount of adriamycin is deposited in the heart [2]. Studies have shown that at a dose of $15.0 \mathrm{mg} / \mathrm{kg}$, adriamycin can cause early oxidative changes in the heart [5] and congestive heart failure [6]. Another study indicated that cardiac changes were not associated with the metabolites of adriamycin after $6.0 \mathrm{mg} / \mathrm{kg}$ intravenous administration or $10 \mathrm{mg} / \mathrm{kg}$ intraperitoneal injection [2]. Is the degree of heart injury associated with kidney injury at different doses? Is there any endogenous molecule that can act as a messenger for heart-kidney interaction? These are questions worth exploring.

Metabolomics is a rapidly evolving biochemical method employed in the analytical field. It has been increasingly applied to basic research fields of foods and phar- maceuticals, such as disease classification, marker identification, new drug development, and microbiology [7-9]. In this study, we used metabolomics to mine the intrinsic cardiorenal metabolic communication in an adriamycininduced nephropathy model.

\section{Materials and Methods}

\section{Materials and Reagents}

Adriamycin was purchased from Shenzhen WanLe Pharmaceutical Co. Ltd. High-performance liquid chromatography-grade formic acid and ethanol were acquired from Fisher Scientific (Fair Lawn, NJ, USA). Pure distilled water was purified using the Millipore Milli-Q water purification system (Bedford, MA, USA). All other reagents used were of at least analytical grade.

\section{Experimental Animals}

Fourteen male-specific pathogen-free Sprague Dawley rats (weight, $180 \pm 20$ g; animal certificate No. SCXK [shaanxi] 2018001, No. 204) were purchased from the experimental animal center of Xi'an Jiaotong University. The animals were maintained at 12-h light/dark illumination periods. The indoor temperature and humidity were $23^{\circ} \mathrm{C} \pm 1.5^{\circ} \mathrm{C}$ and $45 \% \pm 15 \%$, respectively. After 7 days of acclimatization, the rats were randomly categorized into a control group and a model group. On the first day of the experiment, the model group rats were injected with adriamycin $(6.5 \mathrm{mg} / \mathrm{kg})$ via the tail vein [10], and the control group rats were injected with normal saline. On the 20th day after adriamycin administration, the rats were placed alone in a metabolic cage, and urine samples were collected in $24 \mathrm{~h}$. The rats were anesthetized by intraperitoneal injection of chloral hydrate $(300 \mathrm{mg} / \mathrm{kg})$, and $2.5 \mathrm{~mL}$ of inferior venous blood was collected and centrifuged at $-4^{\circ} \mathrm{C}$ at 3,500 rpm for $10 \mathrm{~min}$; the supernatant was collected and stored at $-80^{\circ} \mathrm{C}$. Finally, the rats were euthanized, and the heart and bilateral kidneys were excised. Approximately $1 \mathrm{~cm}^{3}$ of tissues was cut off and fixed in $4 \%$ paraformaldehyde overnight for pathological analysis. The remaining tissues were stored at $-80^{\circ} \mathrm{C}$. The levels of the biochemical indicators serum albumin (ALB), serum globulin (GLOB), serum total protein (TP), and serum total cholesterol (TC) were determined using the Hitachi 7060 automatic biochemical analyzer (Hitachi, Japan). Urinary protein (UPRO) content was determined using Coomassie brilliant blue staining. The ALB/ GLOG $(\mathrm{A} / \mathrm{G})$ ratio was calculated by dividing the ALB level by the GLOB level. The serum creatinine (SCr) and urinary creatinine (UCr) levels were measured using the Creatinine Assay Kit (Jiancheng Biotechnology). The creatinine clearance rate was calculated using the standard formula: creatinine clearance $=(\mathrm{UCr}$ $[\mu \mathrm{mol} / \mathrm{L}] \times 24 \mathrm{~h}$ urine output $[\mathrm{mL}]) /(\mathrm{Scr}[\mu \mathrm{mol} / \mathrm{L}] \times 1,440[\mathrm{~min}] \times$ rat body weight $[\mathrm{kg}]$ ).

\section{Histopathological Examination}

Heart and kidney tissue samples were embedded in paraffin, dewaxed, rehydrated, and stained with hematoxylin-eosin (HE) using a previously described method [11]. Blinded, semiquantitative evaluation was performed to assess kidney and heart pathology. The injury scores were calculated following previously described protocols $[12,13]$. 
A
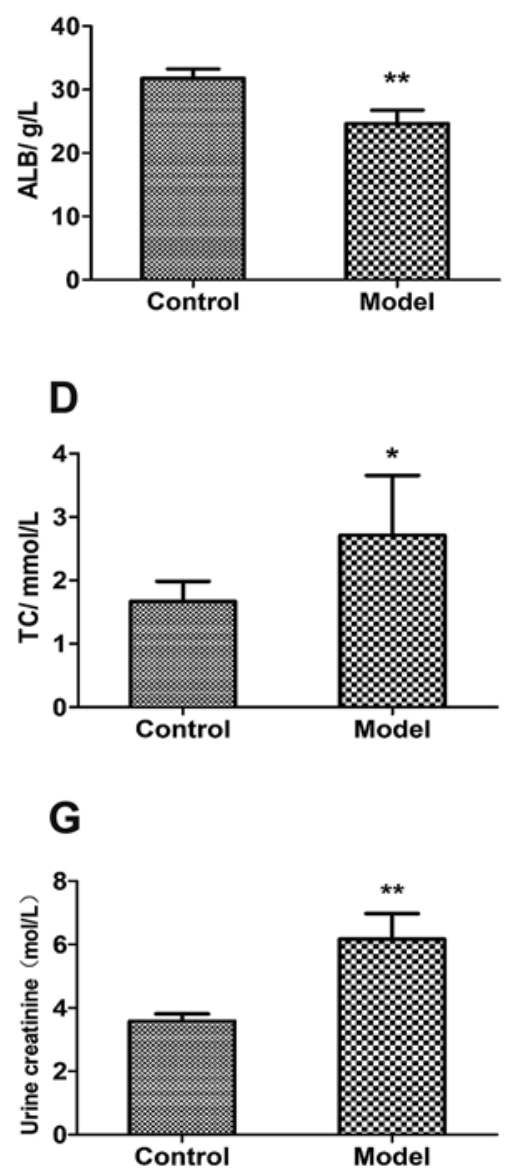

\section{B}
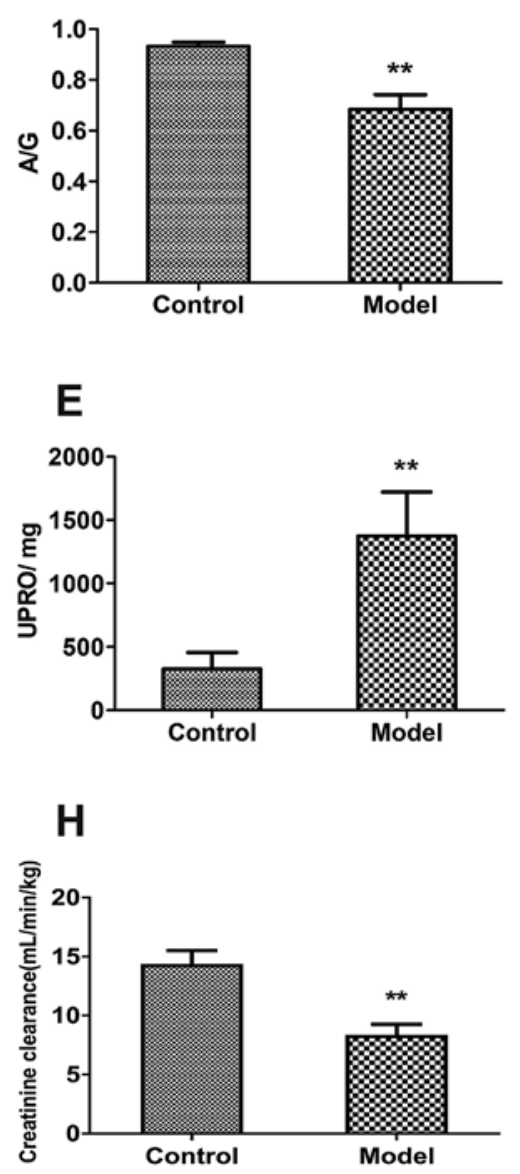

\section{C}
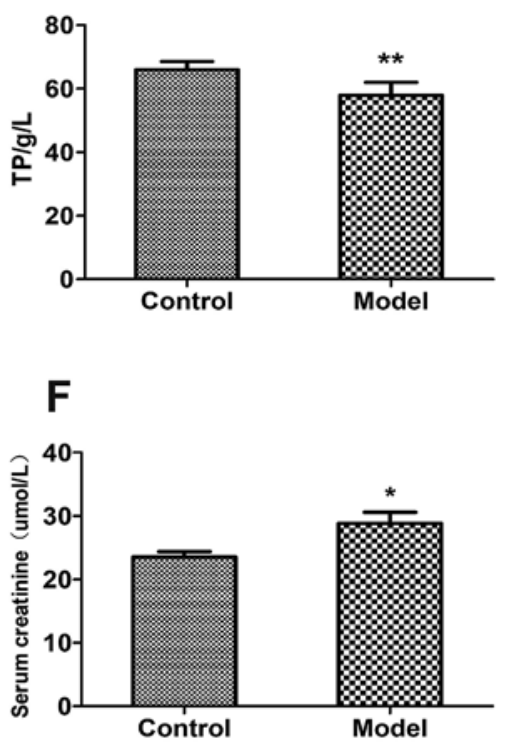

Fig. 1. Rats received a single $6.5-\mathrm{mg} / \mathrm{kg}$ tail vein injection of adriamycin. On the 21 st day, serum ALB (A), A/G ratio (B), and TP (C) were significantly decreased; TC (D) and UPRO (E) were significantly increased; SCr $(\mathbf{F})$ and $\mathrm{UCr}(\mathbf{G})$ were significantly increased; and the creatinine clearance rate $(\mathbf{H})$ was significantly decreased. ${ }^{*} p<$ $0.05,{ }^{* *} p<0.01$. ALB, albumin; A/G, albumin/globulin; TP, total protein; TC, total cholesterol; UPRO, urinary protein; $\mathrm{SCr}$, serum creatinine; UCr, urinary creatinine.

Plasma, Heart, and Kidney Sample Preparation

A total of $200 \mu \mathrm{L}$ of plasma was mixed with $600 \mu \mathrm{L}$ of methanol, vortexed and centrifuged for $3 \mathrm{~min}$ at $6,000 \mathrm{rpm}$. The supernatant was collected, and the substratum was mixed with an additional 400 $\mu \mathrm{L}$ of methanol, vortexed, and centrifuged for $3 \mathrm{~min}$ at 12,000 rpm. The supernatant was then mixed with the previously obtained supernatant and dried under nitrogen gas before reconstituting in $200 \mu \mathrm{L}$ of methanol. The heart and kidney tissues of the rats were thawed at room temperature and homogenized in saline solution $(1: 2, w / v)$. Tissue-grinding fluid was treated in the same way as plasma.

\section{Ultra-Performance Liquid Chromatography/Tandem MS}

Analysis

Separation via liquid chromatography was performed on a Hypersil GOLD C ${ }_{18}(100 \mathrm{~mm} \times 2.1 \mathrm{~mm}, 1.9 \mu \mathrm{m})$ chromatographic column at $35^{\circ} \mathrm{C}$ at a flow rate of $0.3 \mathrm{~mL} / \mathrm{min}$. The injection volume was $3 \mu \mathrm{L}$, and the mobile phase comprised methanol (A) and $0.1 \%$ formic acid in water (B). The solvent gradient system was set as follows: $0-10 \mathrm{~min}, 2 \%$ phase A; $10-30 \mathrm{~min}, 2-80 \%$ phase $\mathrm{A} ; 30-45$ min, $80-90 \%$ phase A; and $45-55$ min, $90-2 \%$ phase A. Mass spectrometry (MS) analysis was performed on a Thermo Scientific QExactive hybrid quadrupole-Orbitrap mass spectrometer equipped with an electrospray ionization source (Thermo Fisher, Waltham, MA, USA). The spray voltages were $3.5 \mathrm{kV}$ in the positive mode and $2.5 \mathrm{kV}$ in the negative mode. The switching mode of positive and negative ions was adopted, with full-scan/dd-MS ${ }^{2}$ mode from 50 to $750 \mathrm{~m} / \mathrm{z}$. The Orbitrap resolution in MS full scan and MS/MS were 70,000 and 17,500 full width at half maximum, respectively. The collision energies were 20,30, and $50 \mathrm{eV}$. The sheath gas and auxiliary gas flow rates were 35 and 15 Arb, respectively. The temperature of the ion transfer tube was $350^{\circ} \mathrm{C}$, and the vaporizer temperature was $300^{\circ} \mathrm{C}$. 
A

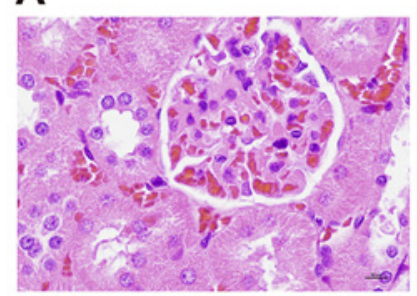

E
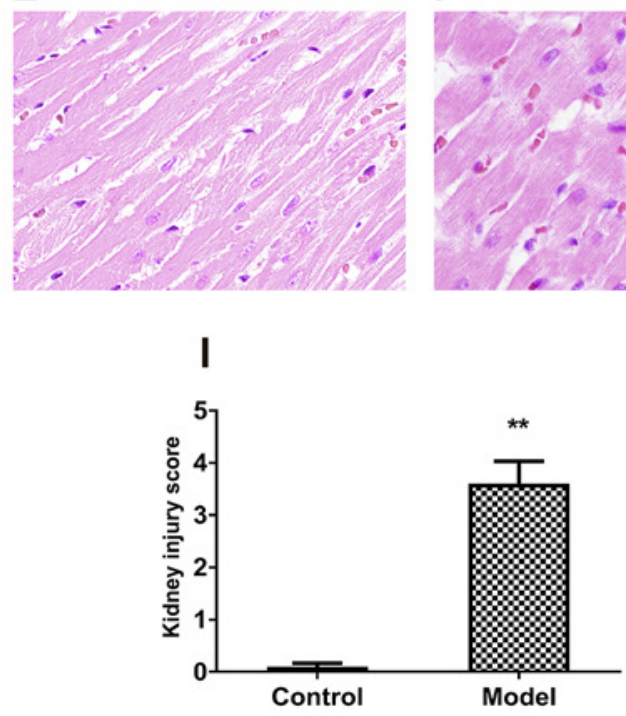

Fig. 2. Significant pathological changes occurred in the heart and kidney tissues. A, B Representative visual fields of the renal tubules and glomeruli in the control group. C, D Representative visual fields of the renal tubules and glomeruli in the model group. $\mathbf{E}, \mathbf{F}$

\section{Data Analysis}

We performed an independent $t$-test to examine betweengroup comparisons. An unpaired parametric $t$-test was performed for between-group comparisons of ALB, A/G, TP, TC, UPRO, Scr, $\mathrm{UCr}$, and creatinine clearance rate. A nonparametric statistical test (Kolmogorov-Smirnov test) was performed for between-group comparisons of the kidney and heart semiquantitative scores (SQSs).

The original MS data files were imported into Compound Discoverer software to build an array comprising retention time and peak area data. Mean-centering normalization, Pareto scaling, and multivariate data analysis were conducted using the MetaboAnalyst 4.0 web-based analytical pipeline. Principal component analysis (PCA) and orthogonal projections to latent structures discriminant analysis (OPLS-DA) were performed [14-16]. A $t$-test was performed to determine the statistical differences in the variables among the groups. Variable important in projection (VIP) $>1.0$ and $p$ value $<0.05$ were set as the thresholds for differential metabolite screening. Based on the analysis module of MetPA, potential metabolic pathways were identified in the heart, plasma, and kidney.
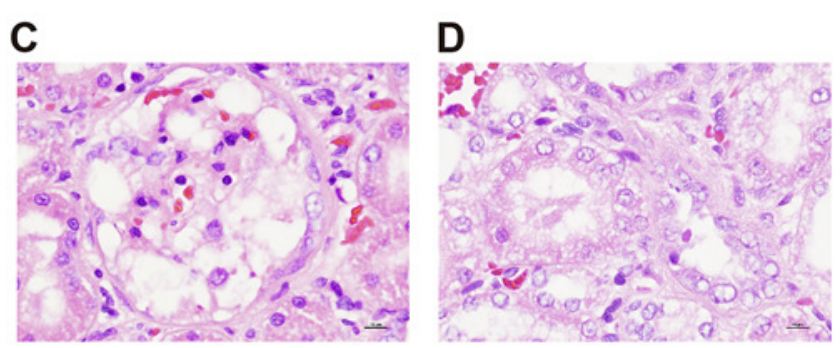

G
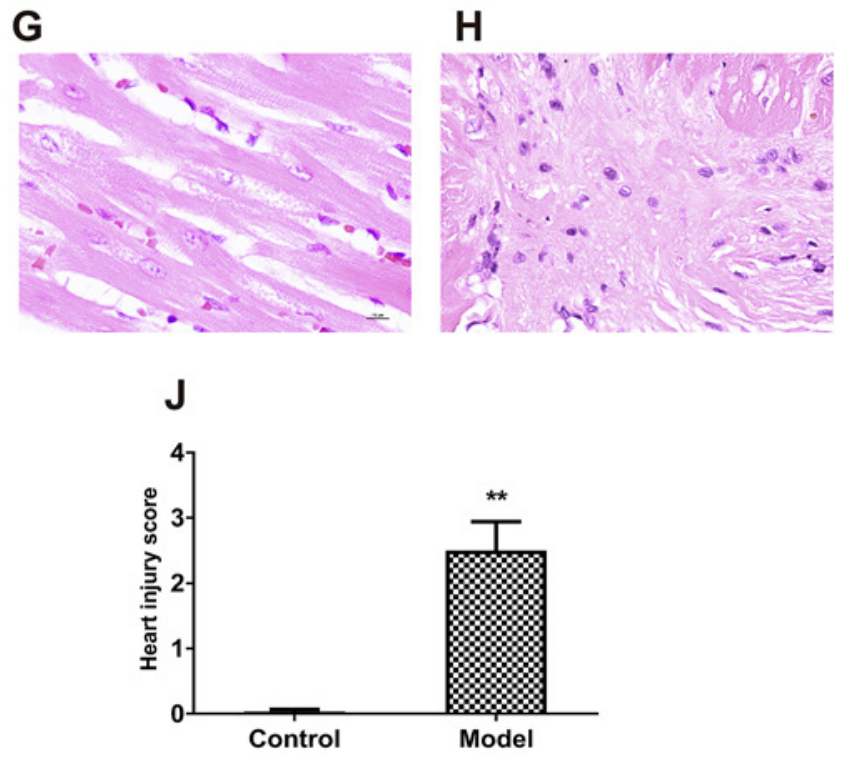

Representative visual fields of the myocardium in the control group. G, H Representative visual fields of the myocardium in the model group. I, J Results of the semiquantitative statistical analysis of the kidneys and heart, respectively. ${ }^{*} p<0.05,{ }^{* *} p<0.01$.

\section{Results}

\section{Serum Biochemical Factors and Histopathological Changes}

As shown in Figure 1A-H, compared with the control group, in the model group, the contents of ALB, A/G, TP, and creatinine clearance rate significantly decreased, whereas the TC, UPRO, Scr, and UCr of the model group was significantly increased.

Figure 2A-D show the representative fields of $\mathrm{HE}$ staining for kidney histopathological sections. There was no obvious pathological change in the control group. In the model group, glomerular epithelial cells were diffusely merged and vacuolated, the number of glomerular nuclei was decreased, and some areas of the glomerulus were sclerotic (Fig. 2C). Renal tubular cells were greatly reduced in size, vacuolated, and accompanied by inflamma- 


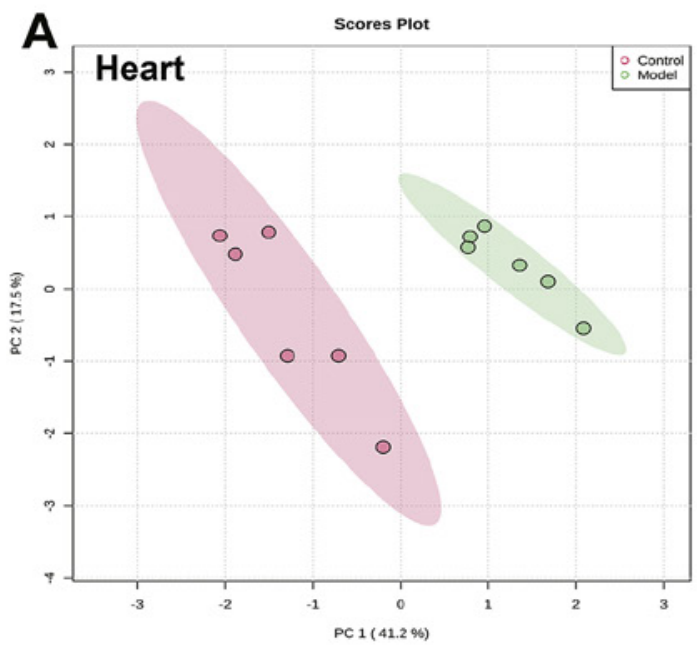

C

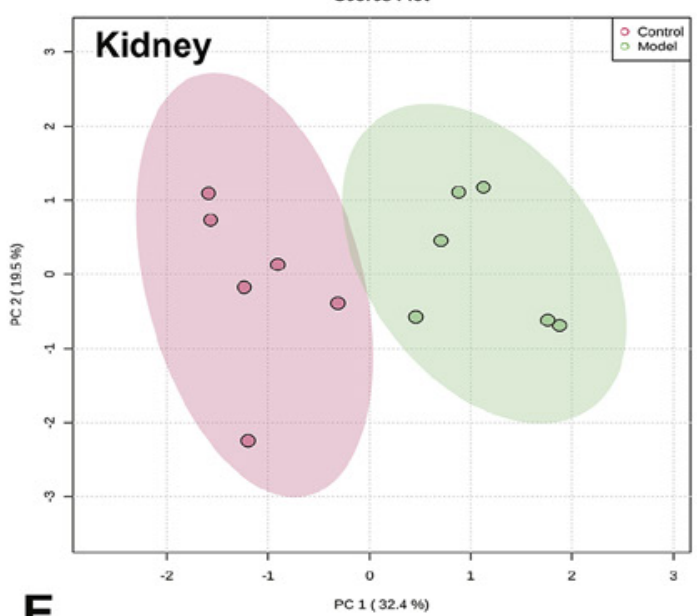

E

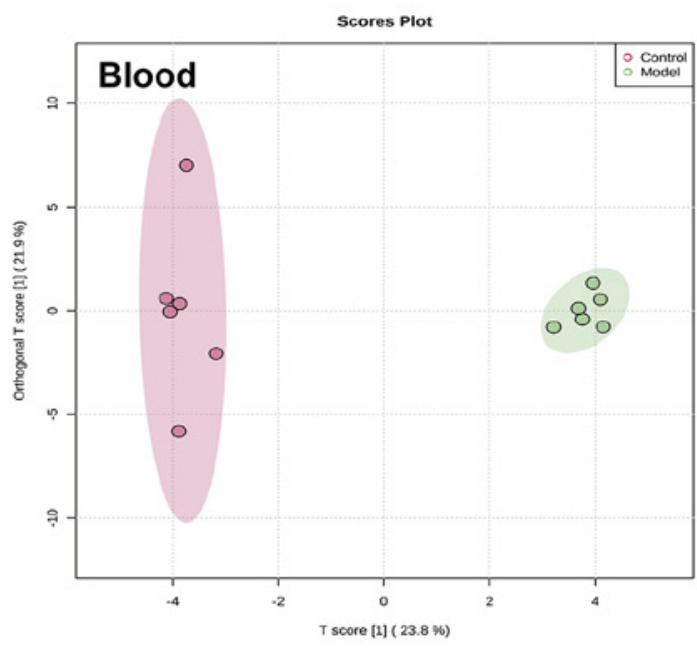

B

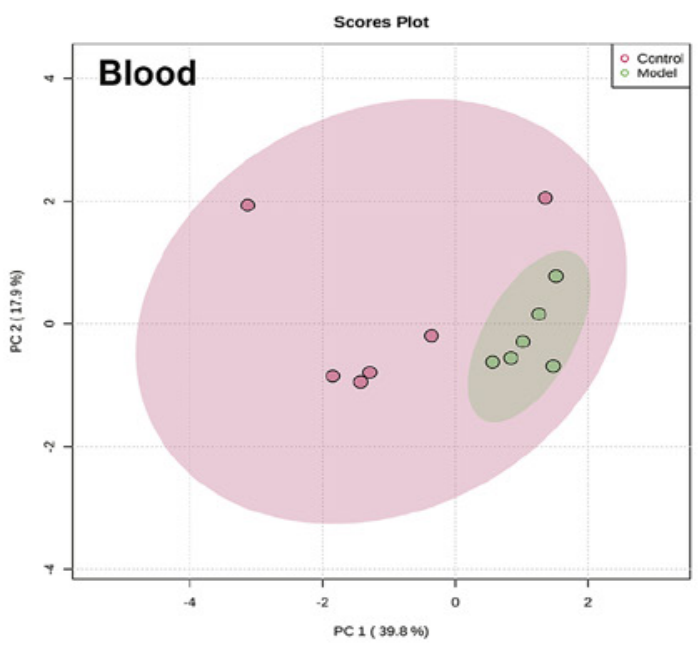

D
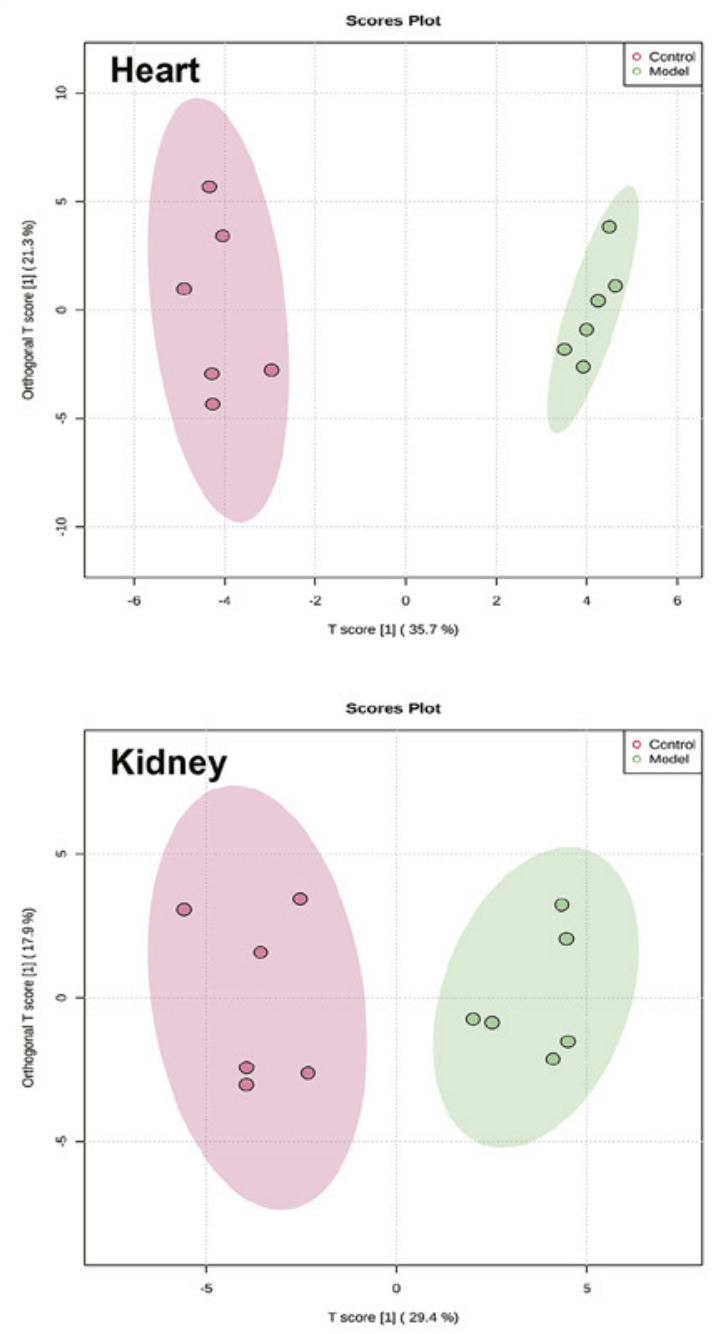

Fig. 3. Multivariate statistical analysis. A-C Results of PCAfor heart, blood, and kidney samples. D-F Partial least squares discrimination analysis for heart, blood, and kidney samples. PCA, principal component analysis 


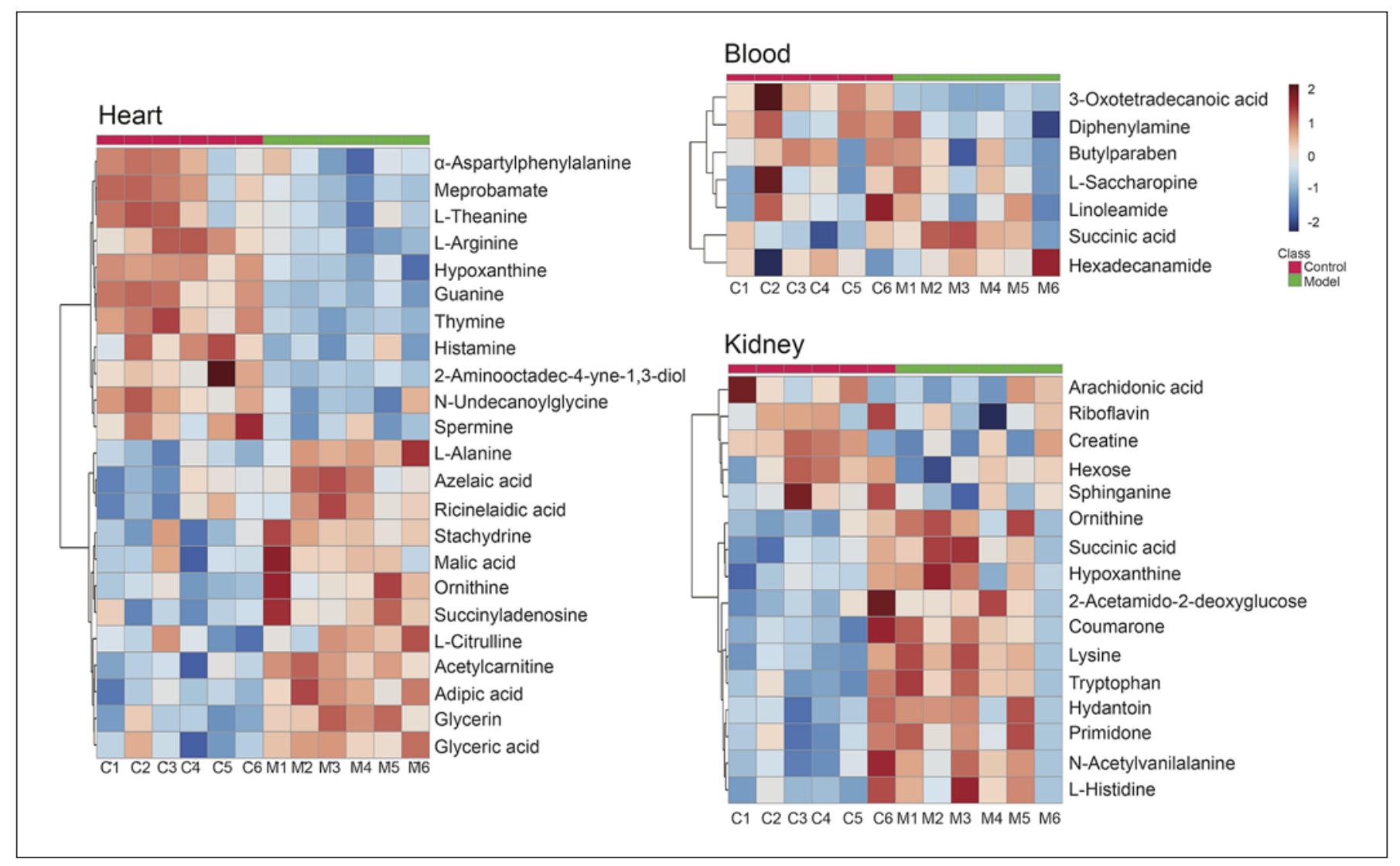

Fig. 4. Heat map of the differential metabolites in the blood, heart, and kidney. The color of the filled squares indicates the normalized intensity values of each metabolite.

tory infiltration (Fig. 2D); Figure 2I shows the overall SQS results. Compared with that in the control group, SQS in the model group significantly increased to $3.6 \pm 1.1$ ( $p<$ 0.01 ). The HE staining results of heart pathological sections are shown in Figure 2E-H. No obvious pathological change was observed in the control group. In the model group, thickened myocardial fibers, swollen nuclei, and inflammatory infiltration could be observed (Fig. 2G), and parts of the myocardial cells began to undergo necrosis (Fig. 2H). Compared with that in the control group, in the model group, SQS was significantly increased to $2.5 \pm$ $1.1(p<0.01$; Fig. 2J). Online suppl. Fig. 1-4 (see www. karger.com/doi/10.1159/000519015 for all online suppl. material) show the other pathological pictures.

\section{Differential Metabolites in the Heart, Blood, and Kidneys}

Online suppl. Fig. 5 shows the total ion chromatograms of the heart, blood, and kidney samples obtained in electrospray ionization-positive and -negative ion modes.
PCA and OPLS-DA analysis were utilized to assess the overall metabolic profiles of the samples. Figure 3 shows the PCA and OPLS-DA analysis diagrams. According to Figure $3 \mathrm{~A}-\mathrm{C}$, the heart, blood, and kidney samples in the control and model groups were clearly divided into 2 clusters in the plane of the first and second principal component axes. The OPLS-DA model was established to magnify the slight difference between the 2 groups. Compared with those in the control group, the metabolites in the plasma, heart, and kidneys of the model group significantly changed, as shown in Figure 3D-F. Model parameters of R2Y and Q2 were used to evaluate the reliability of the OPLS-DA model. The R2Ys of the heart, blood, and kidneys were $0.932,0.698$, and 0.877 , respectively. The Q2s of the heart, blood, and kidneys were $0.839,0.542$, and 0.745 , respectively. All parameters were $>0.50$, indicating that all the OPLS-DA models shown in Figure 3 are reliable. Combined with the results of the $t$-test from MetaboAnalyst 4.0, 20 differential metabolites were identified in the heart of the model group (VIP $>1, p<0.05$ ), 7 were identi- 

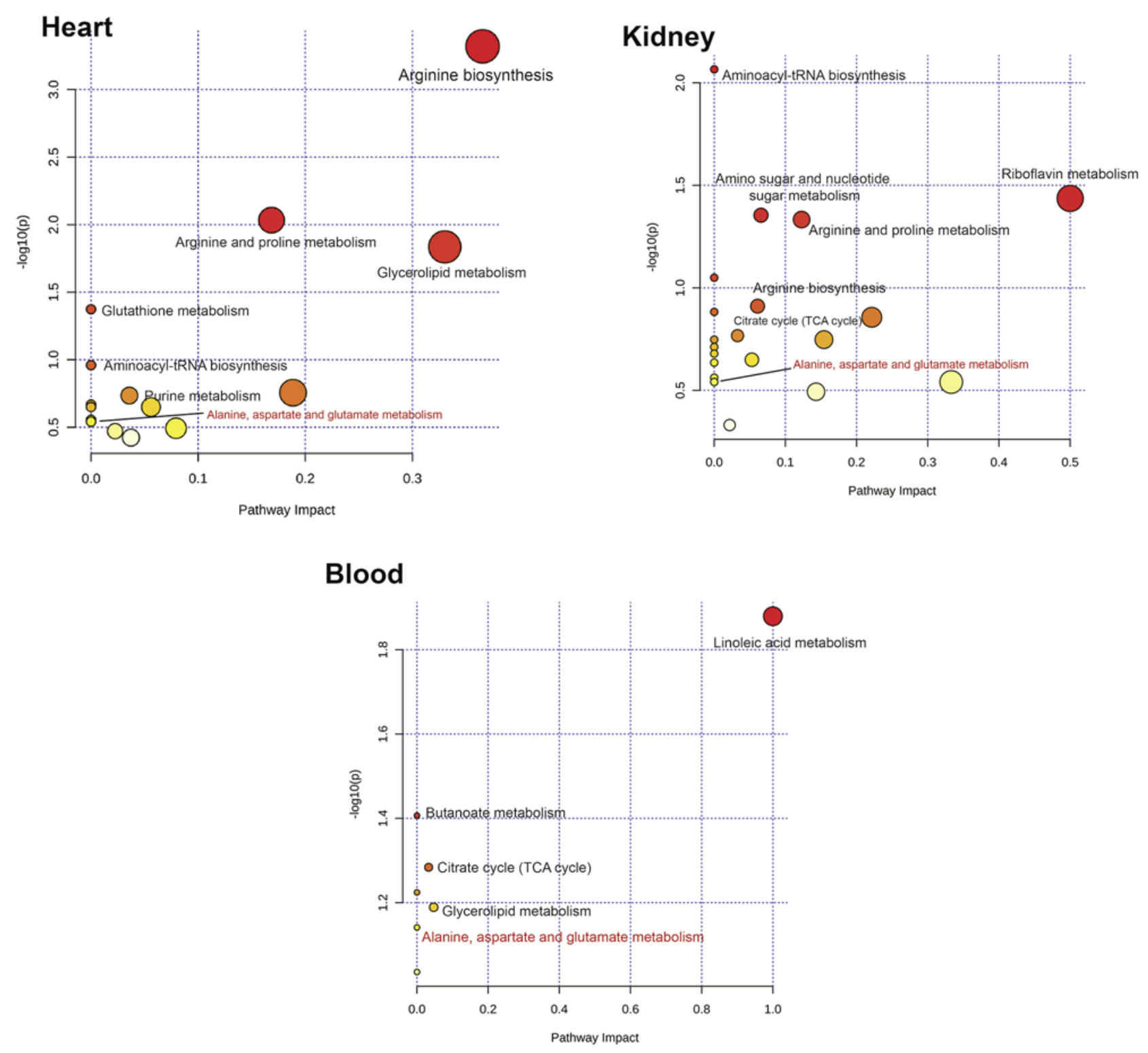

Fig. 5. Pathway analysis of the identified metabolites in the heart, blood, and kidney. Pathways were enriched by MetPA.

fied in the blood (VIP $>1, p<0.05$ ), and 16 were identified in the kidneys (VIP $>1, p<0.05$ ). Online suppl. Table 1 shows the specific information of the metabolites. A heat map was constructed to visualize the relative peak intensities of the differential metabolites in the heart, blood, and kidneys (Fig. 4). Guanine, thymine, and glycerin were most notably changed in the heart (VIP $>1.5$; online suppl. Table 1). All 7 metabolites in the blood were remarkably changed (VIP $>1.5$ ). Similarly, 5 metabolites were changed notably (VIP >1.5) in the kidneys. The ornithine level was increased in the heart and kidneys simultaneously, whereas the succinic acid level was elevated in the blood and kidneys simultaneously (online suppl. Table 1; Fig. 4).

Changes in Metabolic Pathways in the Heart, Blood, and Kidneys

According to MetaboAnalyst 4.0, differential metabolites associated with 15, 7, and 21 metabolic pathways were 
A

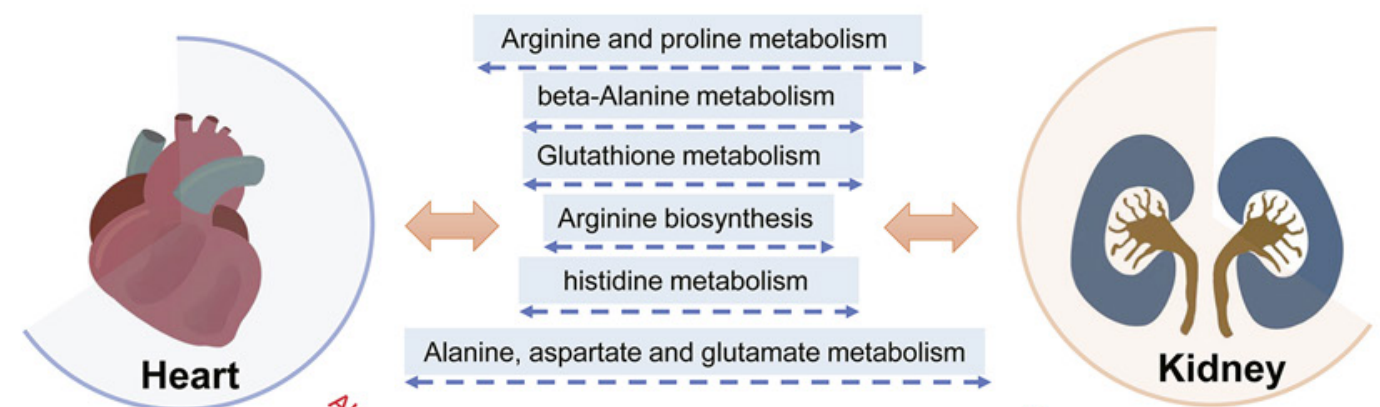

B

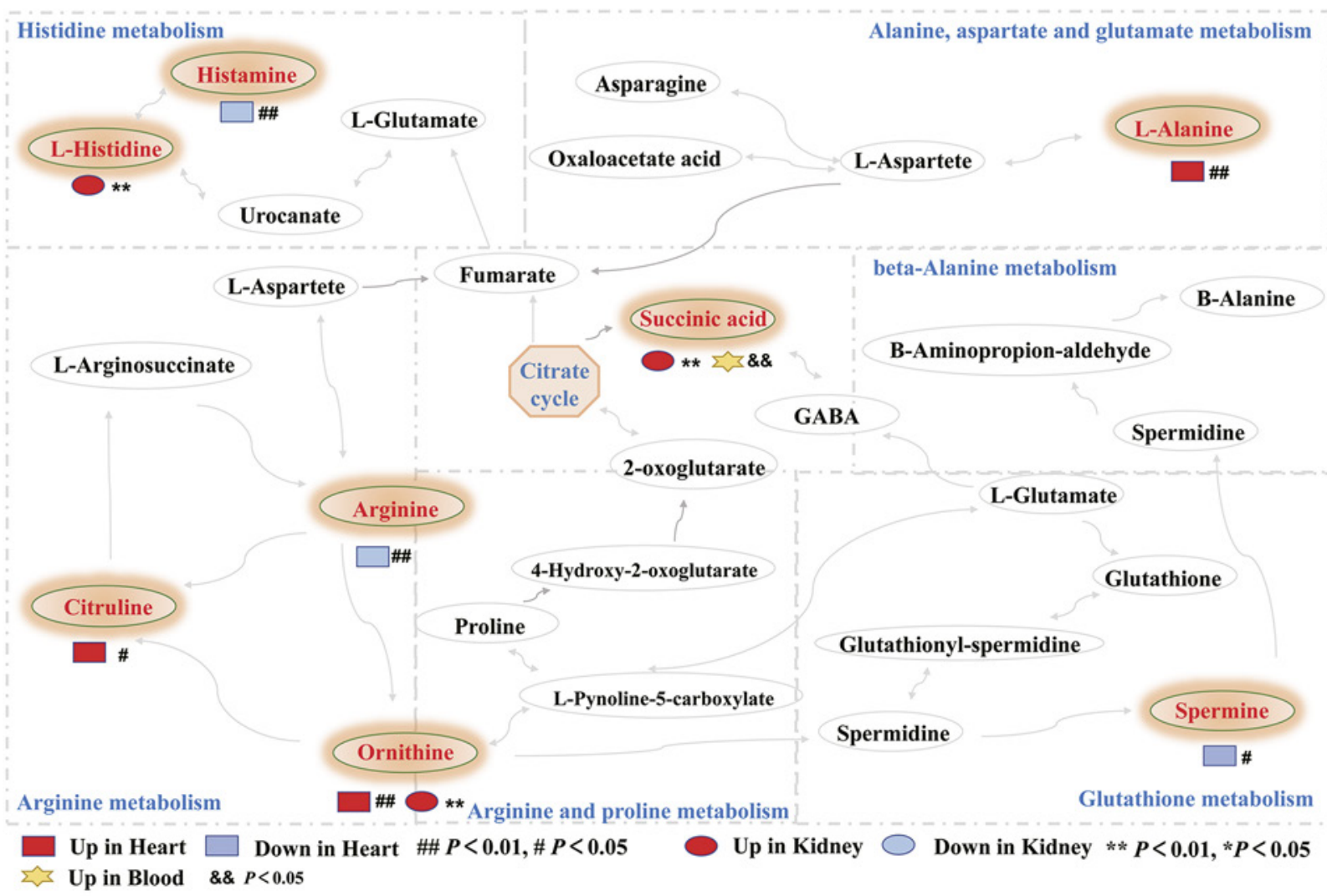

Fig. 6. Oxidative stress and inflammation-related metabolic pathways (A) and corresponding differential metabolites (B) in the heart, kidney, and blood in rats with cardiac and renal co-injury. 
identified in the heart, blood, and kidneys, respectively. Figure 5 shows the main metabolic pathways. As shown in Figure 5, differential metabolic pathways that involved more than 2 differential metabolites in the heart were arginine metabolism, arginine and proline metabolism, glycerolipid metabolism, glutamate metabolism, etc. The important metabolic pathways in the kidneys were aminoacyl-tRNA biosynthesis; riboflavin metabolism; amino sugar and nucleotide sugar metabolism; arginine and proline metabolism; arginine biosynthesis; histidine metabolism; citrate cycle (TCA cycle); alanine, aspartate, and glutamate metabolism; etc. The disrupted metabolic pathways in the blood included linoleic acid metabolism; butanoate metabolism; TCA cycle; propanoate metabolism; lysine degradation; and alanine, aspartate, and glutamate metabolism, whereas linoleic acid metabolism, and unsaturated fatty acid biosynthesis belong to lipid metabolism. TCA cycle, butanoate metabolism, and propanoate metabolism belong to carbohydrate metabolism. Lysine degradation and alanine, aspartate, and glutamate metabolism belong to amino acid metabolism.

The primary focus of this study was the metabolic relationship between the heart and kidneys under simultaneous cardiac and renal injury. Because excessive oxidative stress and inflammation are the main mechanisms during the progression of heart and kidney injury, common pathways related to oxidative stress and inflammation were selected for separate discussion. As shown in Figure 6A, 6 metabolic pathways shared by the heart and kidney were beta-alanine metabolism, arginine biosynthesis, arginine and proline metabolism, glutathione metabolism, aminoacyl-tRNA biosynthesis, and histidine metabolism. The only common metabolic pathway shared by the heart, blood, and kidneys simultaneously was alanine, aspartate, and glutamate metabolism.

Figure 6B shows the corresponding differential metabolites involved in the differential metabolic pathways. Both arginine biosynthesis and arginine and proline metabolism were enhanced in the heart and kidneys. Ornithine levels were increased in both heart and kidneys, whereas citrulline levels were increased only in the heart. Histidine metabolism was enhanced in the kidneys, whereas histamine metabolism was decreased in the heart. The significant increase in alanine and decrease in arginine occurred simultaneously in the heart, indicating that arginine may be converted to alanine. Additionally, it could be easily seen that succinic acid was increased in the kidney and blood simultaneously, implying that energy metabolism changed in the same way between the kidney and blood.

Cardiorenal Metabolic Communication

\section{Discussion}

The adriamycin-induced NS model is primarily characterized by high proteinuria, hypoalbuminemia, and hyperlipidemia. Tubular interstitial lesions started from the 7 th day, were moderated on the 14th day, and worsened on the 21st-28th days. The microscopic pathological development of the kidney progresses from early podocyte injury to interstitial inflammatory infiltration, renal tubular hypertrophy, and collagen deposition (fibrosis), and gradually develops into mesangial matrix deposition and glomerular sclerosis. In this study, an adriamycininduced NS model was established by a one-time injection of adriamycin via the tail vein [2]. On the 21 st day, significant changes in serum biochemical indicators were detected, TC and UPRO of the model rats were significantly increased, and ALB, A/G, and TP were significantly decreased. In addition, Scr and UCr were significantly increased, and the creatinine clearance rate was significantly decreased. The abnormal performance of the above biochemical indicators shows that the model rats had typical high proteinuria, hypoalbuminemia, and hyperlipidemia and disordered glomerular filtration function. These results are consistent with the findings of a previous report [2]. Semiquantitative pathological analysis showed that degenerative vacuolar glomerulus, renal tubular hypertrophy, inflammation, vacuolation, cell reduction, and other pathological changes in the model group were consistent with the progression of the adriamycin-induced NS model in the literature [2]. In addition, myocardial fiber swelling and necrosis were observed in cardiac pathological lesions. The significant lesions of myocardial tissues were not consistent with the results of a previous study, which reported that at a dose of $6.0 \mathrm{mg} /$ $\mathrm{kg}$, adriamycin metabolites would not cause damage to myocardial tissues [2]. The different results may be due to the different observation periods and the influence of intravenous administration. Nevertheless, the above results indicate that it is extremely necessary to explore the accompanying myocardial damage in the adriamycin-induced NS model.

Study [2] has indicated that the nephrotoxicity caused by adriamycin may be due to the formation of free oxygen radicals and reactive oxygen species (ROS). ROS are also involved in the development of podocyte damage and proteinuria. Additionally, the inflammatory response is another important factor that leads to the progression of NS to end-stage renal disease [2]. We employed metabolomics analysis to analyze the heart, blood, and kidney samples of model and control rats. Most of the differential

Kidney Blood Press Res 2022;47:31-42 DOI: $10.1159 / 000519015$ 
pathways in the kidneys are closely associated with inflammation and oxidative stress. For example, arginine and proline metabolism is the core process of arginine, ornithine, proline, citrulline, and glutamate biosynthesis and metabolism [17]. Arginine is directly related to the production of nitric oxide in the body and participates in the regulation of end-stage renal degeneration [18-20]. In addition, the aminoacyl-tRNA biosynthesis pathway is related to oxidative stress, cell-cycle arrest, and apoptosis [21]. Besides arginine, several core differential metabolites are worth discussing. For example, a high sphingomyelin level may be a risk factor for the initiation and progression of kidney disease and coronary heart disease [22].

Oxidative stress, inflammation, and apoptosis are the basic mechanisms of adriamycin-induced cardiotoxicity. The specific biological processes involved include DNA damage, ROS accumulation, lipid peroxidation, energy deficiency, mitochondrial destruction, lysosome destruction, calcium disorder, and autophagy dysfunction [23]. The disordered metabolic pathways in the heart include arginine biosynthesis, arginine and proline metabolism, glycerolipid metabolism, glutathione metabolism, aminoacyl-tRNA biosynthesis, purine metabolism, ornithine metabolism, etc. Because the strong association of arginine biosynthesis and arginine and proline metabolism with aminoacyl-tRNA biosynthesis and oxidative stress has been mentioned in the last section of this article, these pathways are not discussed further here. The representative metabolites in the heart are glycerol, L-arginine, Lalanine hypoxanthine, guanine, etc. Glycerol is related to lipid metabolism and ornithine metabolism. L-arginine and L-alanine are related to aminoacyl-tRNA biosynthesis, which has important physiological functions in the body. For patients with cardiopulmonary failure, glycerolipid metabolism, aminoacyl-tRNA biosynthesis, and glutathione metabolism are all related metabolic pathways implicated in oxidative stress, lactic acidosis, and mitochondrial dysfunction [24]. In addition, arginine plays a key role in regulating cardiovascular dysfunction [25-28]. Both hypoxanthine and guanine are related to purine metabolism. Cardiac hypertrophy and heart failure were shown to be associated with changes in purine nucleotide metabolism $[29,30]$.

To determine the metabolic communication between the heart and kidneys, an alignment of the disrupted pathways was performed between the 2 organs. Ten common disordered metabolic pathways were identified, 6 of which were related to oxidative stress and inflammation, including arginine and proline metabolism [31]; arginine biosynthesis [32]; glutathione metabolism [33]; alanine, aspartate, and glutamate metabolism; beta-alanine metabolism [34]; and histidine metabolism [35]. We also noted that the change in the direction of the same metabolic pathways in the heart, blood, and kidneys was partially different. In addition to an increase in succinic acid, a significant elevation of histidine was also observed in the kidneys. Histidine is a precursor of antioxidant dipeptide. Elevated histidine and succinic acid levels represent the overall respiration of activated energy metabolism and antioxidant systems in the kidneys. Compared with the metabolic difference in the kidney, the metabolic difference in the heart was presented in 2 aspects: the decreased histamine level and the accumulation of the spermine precursors ornithine and citrulline. Spermine is one of the polyamines in the body and is considered to be closely involved in cellular functions. The spermine level is usually abnormally elevated in tissue and cells with inflammation or abnormal energy metabolism. Based on our results, the decrease in spermine levels in the heart might be due to a compensatory regulation induced by the conversion of arginine to alanine. The complicated mechanisms that exist between histamine and spermine have not yet been elucidated. Available evidence can prove only that injection of exogenous spermine could stimulate the release of histamine [36]. Whether the simultaneous decrease in spermine and histamine is the cause or the consequence of the interaction requires further exploration. Another interesting result was the comparison of the decrease in histamine levels in the heart and increase in histidine levels in the kidneys. Based on the heart-kidney link with blood circulation, we presumed that histamine in the heart may serve as the raw material for histidine synthesis in the kidneys through blood circulation. Further studies are needed to fully explore the exact mechanism. Overall, the 6 metabolic pathways that were common between the heart and kidneys in this study may offer clues that reveal the metabolic heart-kidney communication.

Because both the heart and kidneys possess rich blood flow, it is speculated that there might be metabolic pathways in the blood that serve as the bond between cardiac and renal injury pathological processes. Alanine, aspartate, and glutamate metabolism was found to be the common disrupted pathway in the kidney, heart, and blood. Hence, a natural conjecture is whether alanine, aspartate, and glutamate metabolism is one of the metabolic links between co-injury of the heart and kidneys. Several studies have provided clues about this: alanine, proline, and arginine metabolism can predict proteinuria progression,
Wang/Liang/Yang/Wang/Yu/Jia/Du/ Wang/Li/Zheng 
beta-alanine metabolism, arginine and proline metabolism, and TCA cycle and is closely associated with proteinuria, progression of renal damage, and hypertension [37]. The abovementioned studies provide supporting evidence for the importance of alanine, aspartate, and glutamate metabolism in the metabolic communication between the heart and kidneys in simultaneous cardiac and renal injury. However, nontargeted metabolomics can provide only metabolic profiling and part of the differential metabolites involved in alanine, aspartate, and glutamate metabolism pathways. More comprehensive changes in the key metabolites might be found in future studies using targeted metabolomics.

The present study results show that there is one metabolite in alanine, aspartate, and glutamate metabolism that might be valuable for further research. Succinic acid may be the most prominent metabolite that changes most significantly in the blood. Succinic acid is an important intermediate of alanine, proline, and arginine metabolism and the TCA cycle. High-energy electron-containing flavin adenine dinucleotide generated by succinic acid oxidation can enter the electron transport chain from different sites and is involved in ROS generation in the mitochondria. As demonstrated experimentally, treatment with sodium succinate significantly decreased ROS levels and improved renal function $[18,38]$. As a key metabolic small molecule involved in respiratory chain and mitochondrial oxidation, the real-time succinic acid level in tissues and blood may have a key indicative value for the degree of oxidative stress damage in the heart and kidneys $[18,38]$. Moreover, studies have suggested that succinate also acts as a metabolite in innate immune signaling to enhance inflammatory factors such as interleukin-1B [39]. Thus, succinic acid may serve as a potential metabolite marker for excessive oxidative stress and inflammation-induced cardiac and renal co-injury.

\section{Conclusion}

Collectively, the metabolic correlation between the heart and kidney was analyzed using a model of adriamycin-induced cardiac and renal co-injury. Arginine and proline metabolism; arginine biosynthesis; glutathione metabolism; alanine, aspartate, and glutamate metabolism; beta-alanine metabolism; and histidine metabolism were found to be the common disordered metabolic pathways between heart and kidney co-injury. In addition, alanine, aspartate, and glutamate metabolism may be the metabolic link between the heart and kidney in the devel- opment and perpetuation of oxidative stress and inflammation. Succinic acid, an important metabolite, may serve as a key regulatory metabolic switch or a marker of cardiac and renal co-injury in adriamycin-induced nephropathy.

\section{Statement of Ethics}

All animal experiment protocols were reviewed and approved by the Animal Management and Ethics Committee, Northwestern University (NWU-AUC-20200601R).

\section{Conflict of Interest Statement}

The authors declare no conflict of interest.

\section{Funding Sources}

This study was financially supported by Program for Changjiang Scholars and Innovative Research in University (IRT_15R55); Project of Key Research and Development Plan of Shaanxi (No. 2017ZDCXL-SF-01-02-01, 2018SF-293), Scientific Research Plan Project of Shaanxi Key Laboratory (No. 15JS108).

\section{Author Contributions}

Chunliu Wang participated in the metabolomic analysis and wrote the manuscript; Jiping Liang, Wenwen Yang, Yapeng Du, Mei Wang, and Shixiang Wang contributed in the animal model preparation and samples process. Jie $\mathrm{Yu}$ and $\mathrm{Pu}$ Jia contributed in the data analysis; Xiaohui Zheng, Pu Jia, and Ye Li participated in the design of the study.

\section{Data Availability Statement}

The data that support the findings of this study are openly available at https://orcid.org/0000-0002-2345-4035. Further inquiries can be directed to the corresponding author.

\footnotetext{
References $\quad 1$ Bertani T, Poggi A, Pozzoni R, Delaini F, Sacchi G, Thoua Y, et al. Adriamycin-induced nephrotic syndrome in rats: sequence of pathologic events. Lab Invest. 1982;46(1):1623.

2 Pereira Wde F, Brito-Melo GE, de Almeida CA, Moreira LL, Cordeiro CW, Carvalho TG, et al. The experimental model of nephrotic syndrome induced by doxorubicin in rodents: an update. Inflamm Res. 2015 May 64;64(5): 287-301.
} 
3 Li AP, Yang L, Zhang LC, He SS, Jia JP, Qin $\mathrm{XM}$. Evaluation of injury degree of adriamycin-induced nephropathy in rats based on serum metabolomics combined with proline marker. J Proteome Res. 2020 Jul 19;19(7): 2575-84.

4 Tan J, Wang J, Geng L, Yue Y, Wu N, Zhang Q. Comparative Study of fucoidan from saccharina japonica and its depolymerized fragment on adriamycin-induced nephrotic syndrome in rats. Mar Drugs. 2020 Mar;18(3): 137.

5 Li T, Singal PK. Adriamycin-induced early changes in myocardial antioxidant enzymes and their modulation by probucol. Circulation. 2000 Oct 102;102(17):2105-10.

6 Tong J, Ganguly PK, Singal PK. Myocardial adrenergic changes at two stages of heart failure due to adriamycin treatment in rats. Am J Physiol. 1991 Mar 260;260(3 Pt 2):H909-16.

7 Eicher T, Kinnebrew G, Patt A, Spencer K, Ying K, Ma Q, et al. Metabolomics and multiomics integration: a survey of computational methods and resources. Metabolites. 2020 May 10;10(5):202.

8 Krzastek SC, Smith RP, Kovac JR. Future diagnostics in male infertility: genomics, epigenetics, metabolomics and proteomics. Transl Androl Urol. 2020 Mar 9;9:S195-205.

9 Lanznaster D, Veyrat-Durebex C, Vourc'h P, Andres CR, Blasco H, Corcia P. Metabolomics: a tool to understand the impact of genetic mutations in amyotrophic lateral sclerosis. Genes. 2020 May11;11(5):537.

10 Chen $\mathrm{KH}$, Chen $\mathrm{CH}$, Wallace CG, Chen YT, Yang CC, Sung PH, et al. Combined therapy with melatonin and exendin-4 effectively attenuated the deterioration of renal function in rat cardiorenal syndrome. Am J Transl Res. 2017 sep 9;9(2):214-29.

11 Feng Y, Guo F, Xia Z, Liu J, Mai H, Liang Y, et al. Inhibition of fatty acid-binding protein 4 attenuated kidney fibrosis by mediating macrophage-to-myofibroblast transition. Front Immunol. 2020 Sep 30;11:566535.

12 Neumaier HL, Harel S, Klingel K, Kaya Z, Heuser A, Kespohl M, et al. ONX 0914 lacks selectivity for the cardiac immunoproteasome in coxsackievirusB3 myocarditis of NMRI mice and promotes virus-mediated tissue damage. Cells. 2020 May 9;9(5):1093.

13 Wang J, Ishfaq M, Fan Q, Chen C, Li J. 7-Hydroxycoumarin attenuates colistin-induced kidney injury in mice through the decreased level of histone deacetylase 1 and the activation of Nrf2 signaling pathway. Front Pharmacol. 2020 Jul 28;11:1146.

14 Gao K, Yang R, Zhang J, Wang Z, Jia C, Zhang F, et al. Effects of Qijian mixture on type 2 diabetes assessed by metabonomics, gut microbiota and network pharmacology. Pharmacol Res. 2018 Apr 130;130:93-109.

15 Cheng P, Xue X, Su J, Lu M, Wang S, Meng J. $1 \mathrm{H}$ NMR-based metabonomic revealed protective effect of Moutan Cortex charcoal on blood-heat and hemorrhage rats. J Pharm Biomed Anal. 2019 May 169;169:151-8.
16 Li W, Hong B, Li Q, Li Z, Bi K. An integrated serum and urinary metabonomic research of Rhizoma Curcumae-Rhizoma Sparganii drug pair in hysteromyoma rats based on UPLCQ-TOF-MS analysis. J Ethnopharmacol. 2019 Mar 231;231:374-85.

17 Majumdar R, Barchi B, Turlapati SA, Gagne M, Minocha R, Long S, et al. Glutamate, ornithine, arginine, proline, and polyamine metabolic interactions: the pathway is regulated at the post-transcriptional level. Front Plant Sci. 2016 Feb 16;7:78

18 Chapela SP, Burgos I, Congost C, Canzonieri $\mathrm{R}$, Muryan A, Alonso M, et al. Parenteral succinate reduces systemic ROS production in septic rats, but it does not reduce creatinine levels. Oxid Med Cell Longev. 2018 Nov 6; 2018:1928945.

19 Hu QD, Wu WH, Zeng Y, Wen J, Li XJ, Pan $\mathrm{W}$, et al. Blood metabolism study on protection of residual renal function of hemodialysis patients by traditional Chinese medicine kidney flaccidity compound. Cell Mol Biol. 2018 May 64;64(5):107-12.

20 Liu S, Liang S, Liu H, Chen L, Sun L, Wei M, et al. Metabolite profiling of feces and serum in hemodialysis patients and the effect of medicinal charcoal tablets. Kidney Blood Press Res. 2018 June 43;43(3):755-67.

21 Liu W, Jin F, Gao D, Song L, Ding C, Liu H. Metabolomics analysis reveals aminoquinazolin derivative 9d-induced oxidative stress and cell cycle arrest in A549 cells. RSC Adv. 2017 Feb 7;7(22):13149-58.

22 Pongrac Barlovic D, Harjutsalo V, Sandholm N, Forsblom C, Groop PH; FinnDiane Study Group. Sphingomyelin and progression of renal and coronary heart disease in individuals with type 1 diabetes. Diabetologia. 2020. Sep; 63:1847-56.

23 Xiao B, Hong L, Cai X, Mei S, Zhang P, Shao $\mathrm{L}$. The true colors of autophagy in doxorubicin-induced cardiotoxicity. Oncol Lett. 2019 Sep 18;18(3):2165-72.

24 Contaifer D Jr, Buckley LF, Wohlford G, Kumar NG, Morriss JM, Ranasinghe AD, et al. Metabolic modulation predicts heart failure tests performance. PLos One. 2019;14(6): e0218153.

25 Popolo A, Adesso S, Pinto A, Autore G, Marzocco S. L-Arginine and its metabolites in kidney and cardiovascular disease. Amino Acids. 2014 Oct 46;46(10):2271-86.

26 Xu YP, Sui XL, Zhang AS, Ye L, Gu FJ, Chen $\mathrm{JH}$. Monocytes, endoplasmic reticulum stress and metabolomics in dogs with multiple organ dysfunction syndrome treated by continuous venovenous hemodiafiltration. Oncotarget. 2017 May 8;8(21):34992-5008.

27 Mao B, Yi Y, Mo Q, Yang C, Zhong Q. Metabolic profiling reveals the heterogeneity of vascular endothelial function phenotypes in individuals at extreme cardiovascular risk. RSC Adv. 2019 Sep 9;9(52):30033-44.
28 Wang H, Liu L, Rao X, Chai T, Zeng B, Zhang $\mathrm{X}$, et al. Commensal microbiota regulation of metabolic networks during olfactory dysfunction in mice. Neuropsychiatr Dis Treat. 2020 Mar 16;16:761-9.

29 Bauer JA, Moffatt-Bruce SD, Elton TS, Feldman D. Purine metabolism in heart failure: oxidant biology and therapeutic indications. Congest Heart Fail. 2008 Sep-oct 14;14(5): 283-4.

30 Liu Y, Yan X, Mao G, Fang L, Zhao B, Liu Y, et al. Metabonomic profiling revealed an alteration in purine nucleotide metabolism associated with cardiac hypertrophy in rats treated with thiazolidinediones. J Proteome Res. 2013 Dec;12(12):5634-41.

31 Toczylowska B, Zieminska E, Senator P, Lazarewicz JW. Hippocampal metabolite profiles in two rat models of autism: NMR-based Metabolomics Studies. Mol Neurobiol. 2020 Jul 57;57(7):3089-105.

32 Gambardella J, Khondkar W, Morelli MB, Wang X, Santulli G, Trimarco V. Arginine and endothelial function. Biomedicines. 2020 Aug 8;8(8):277

33 Ma P, Zhang Z, Zhou X, Luo J, Lu H, Wang Y. Characterizing semen abnormality male infertility using non-targeted blood plasma metabolomics. PLos One. 2019; 14(7): e0219179.

34 Yan JJ, Du GH, Qin XM, Gao L. Baicalein attenuates the neuroinflammation in LPS-activated BV-2 microglial cells through suppression of pro-inflammatory cytokines, COX2/ NF- $\kappa \mathrm{B}$ expressions and regulation of metabolic abnormality. Int Immunopharmacol. 2020 Feb;79:106092.

35 Nasri M, Mahdavifard S, Babaeenezhad E, Adibhesami G, Nouryazdan N, Veiskarami S, et al. Ameliorative effects of histidine on oxidative stress, tumor necrosis factor alpha (TNF-alpha), and renal histological alterations in streptozotocin/nicotinamide-induced type 2 diabetic rats. Iran J Basic Med Sci. 2020 Jun;23(6):714-23.

36 Mizoguchi $\mathrm{H}$, Watanabe $\mathrm{C}$, Hayashi T, Iwata $\mathrm{Y}$, Watanabe H, Katsuyama S, et al. The involvement of spinal release of histamine on nociceptive behaviors induced by intrathecally administered spermine. Eur J Pharmacol. 2017 Apr;800:9-15.

37 Gonzalez-Calero L, Martin-Lorenzo M, Martínez PJ, Baldan-Martin M, Ruiz-Hurtado G, Segura J, et al. Hypertensive patients exhibit an altered metabolism. A specific metabolite signature in urine is able to predict albuminuria progression. Transl Res. 2016 Dec;178: 25-37.e7.

38 Andrienko TN, Pasdois P, Pereira GC, Ovens MJ, Halestrap AP. The role of succinate and ROS in reperfusion injury - a critical appraisal. J Mol Cell Cardiol. 2017 Sep;110:1-14.

39 Tannahill GM, Curtis AM, Adamik J, Palsson-McDermott EM, McGettrick AF, Goel G, et al. Succinate is an inflammatory signal that induces IL-1 $\beta$ through HIF-1 $\alpha$. Nature. 2013 Apr 11;496(7444):238-42. 